\title{
COLONOSCOPIA OU SIGMOIDOSCOPIA: \\ risco de lesões isoladas no cólon direito
}

\author{
Frank Shigueo NAKAO*, Marcelo de Souza CURY* e \\ Angelo Paulo FERRARI Jr.**
}

RESUMO - Racional - Atualmente existem dúvidas quanto ao método ideal de rastreio e vigilância para o câncer colorretal. A retossigmoidoscopia é preconizada, já que é barata, eficaz e causa pouco desconforto, mas não pode diagnosticar tumores do cólon proximal. Objetivo - Avaliar quantas lesões encontradas no cólon proximal seriam perdidas caso a colonoscopia só fosse empregada em pacientes com lesões detectadas durante sigmoidoscopia. Casuística e Método Foram revistas as colonoscopias consecutivas com achado de pólipos ou neoplasia, realizadas no Setor de Endoscopia da Disciplina de Gastroenterologia da Universidade Federal de São Paulo, excluindo pacientes repetidos, operados ou com doença inflamatória intestinal. Resultados - Cento e um exames foram elegiveis, sendo 45 pacientes do sexo masculino. A idade média foi 62 anos ( $D P \pm 13,7$ anos). Cento e oito fragmentos foram enviados separadamente para exame anatomopatológico, sendo 38 com suspeita de neoplasia e 70 pólipos. Ao se considerarem apenas os achados no cólon proximal, observou-se o total de 45 lesões, sendo 23 adenomas, 10 adenocarcinomas, 1 linfoma e 11 lesões benignas. Destas lesões, 28 eram isoladas, sendo 16 adenomas, 7 adenocarcinomas. Conclusão - Observaram-se que 23 pacientes (22,77\%) tinham adenoma ou adenocarcinoma apenas no cólon direito, que não seriam diagnosticados se sigmoidoscopia flexivel fosse usada isoladamente.

DESCRITORES - Colonoscopia. Sigmoidoscopia. Pólipos do cólon. Neoplasias retais.

\section{INTRODUÇÃO}

O câncer colorretal (CCR) é importante causa de morte nas sociedades ocidentais ${ }^{(3)}$ Segundo a Organização Mundial da Saúde, o câncer é a segunda causa de óbito quando excluídas as causas não-naturais. No Brasil, o cólon e reto constituem o quarto local mais freqüente de câncer em homens e quinto em mulheres ${ }^{(5)}$.

O Ministério da Saúde estima, através dos registros de câncer de base populacional, que em 1999 ocorreram 19.050 novos casos de CCR com aproximadamente 6000 mortes $^{(5)}$.
Idade superior a 50 anos, história familiar de CCR, polipose familiar, doença inflamatória intestinal acometendo todo o cólon, radioterapia prévia (abdominal ou pélvica), tabagismo e CCR prévio são os principais fatores de risco ${ }^{(8,10)}$.

Apesar da descrição do câncer "de novo", está bem definida a seqüência adenoma-carcinoma na história natural do $\mathrm{CCR}^{(13,14)}$, sendo a polipectomia reconhecida como profilaxia eficaz ${ }^{(7,12,16)}$. Diagnóstico precoce é a forma mais eficaz para diminuir a mortalidade do $\mathrm{CCR}^{(8)}$.

Diversos esquemas de rastreamento do CCR têm sido propostos para o diagnóstico precoce em pacientes de

Setor de Endocopia Digestiva da Disciplina de Gastroenterologia da Universidade Federal de São Paulo (EPM-UNIFESP)

* Pós-Graduandos.

** Chefe.

Endereço para correspondência: Dr. Marcelo S. Cury - Rua Borges Lagoa, 908/162 - 04038-002 - São Paulo, SP. e-mail: mcury.dmed@unifesp.com.br 
médio risco (idade acima dos 50 anos), como também esquemas de vigilância para os de alto risco (associado a algum dos fatores citados acima) $)^{(1,2,3)}$.

Discute-se atualmente o uso rotineiro da colonoscopia ou da sigmoidoscopia. Embora esta última seja barata e cause pouco desconforto, não pode diagnosticar tumores do cólon proximal.

Este estudo objetiva avaliar quantas lesões encontradas no cólon proximal seriam perdidas caso a colonoscopia só fosse empregada em pacientes com lesões detectadas durante sigmoidoscopia.

\section{MATERIAL E MÉTODO}

Foram revistos os achados de colonoscopias realizadas no período de fevereiro de 1994 a março de 1997, no Serviço de Endoscopia Digestiva da Disciplina de Gastroenterologia da Escola Paulista de Medicina - Universidade Federal de São Paulo (EPM-UNIFESP) e incluídos no estudo os exames de pacientes que apresentavam pólipos ou lesões neoplásicas.

Foram excluídos pacientes com diagnóstico ou suspeita de doença inflamatória intestinal, aqueles submetidos a cirurgia colônica prévia (colectomia, com ou sem colostomia) ou exames de emergência.

Exames que ultrapassassem o ângulo esplênico, nos quais o ceco não foi alcançado não foram excluídos, já que a principal causa foi neoplasia estenosante. Apenas o primeiro exame de pacientes submetidos a mais de uma colonoscopia foi incluído. Foram estudados os pólipos e lesões possivelmente neoplásicas, sua localização e os achados histológicos respectivos.

Consideramos direito ou proximal o segmento do cólon que vai do ceco ao ângulo esplênico, e cólon esquerdo ou distal o segmento que compreende o ângulo esplênico até a borda anal, e que, em geral, está ao alcance da sigmoidoscopia flexível.

\section{RESULTADOS}

De fevereiro de 1994 a março de 1997, pudemos recuperar no arquivo do Setor de Endoscopia 601 colonoscopias. Cento e um exames foram elegíveis para o estudo por apresentarem pólipos e/ou lesões neoplásicas.

Quarenta e cinco foram realizados em pacientes do sexo masculino e 56 do sexo feminino. A idade média foi 62 anos (DP 13,7 anos). Em 22 exames não foi possível a intubação do ceco: 5 por dificuldades técnicas e os outros devido à presença de estenoses. Assim, excluindose a presença de estenose, a colonoscopia foi realizada com sucesso em 96/101 (95\%) exames. Cento e oito fragmentos foram enviados separadamente para exame anatomopatológico, sendo 38 com suspeita de neoplasia e 70 pólipos.

Dos pacientes com suspeita de neoplasia à colonoscopia, 9 $(23,68 \%)$ eram no cólon direito, $28(73,68 \%)$ no esquerdo e 1 apresentou lesões suspeitas tanto no cólon direito, quanto no esquerdo. O diagnóstico histológico deste paciente foi linfoma não-Hodgkin em ambas as lesões. Adenocarcinoma foi confirmado pelo exame histológico em 26 pacientes, sendo que 6 (23\%) eram proximais. Dez das lesões eram adenomas (Tabela 1).

Dentre os 70 pólipos, 34 (48,56\%) eram exclusivamente no cólon esquerdo, 19 (27,14\%) em ambos e 17 (24,28\%) apenas no direito. Cinco pacientes apresentaram múltiplos pólipos e 36 apresentaram um único pólipo. Quanto ao diagnóstico histológico destes pólipos (Tabela 2), observou-se que 48 eram adenomas e 5 adenocarcinomas, sendo 1 no cólon esquerdo e 4 proximais. Na Tabela 3 pode-se observar o tipo histológico dos adenomas encontrados tanto proximais quanto distais.

Ao consideramos apenas os achados no cólon proximal (Tabela 4), observamos o total de 45 lesões, sendo 23 adenomas, 10 adenocarcinomas, 1 linfoma e 11 lesões benignas. Dentre as lesões em cólon proximal, 28 eram isoladas, sendo 16 adenomas, 7 adenocarcinomas. Portanto, 23/101 (22,77\%) pacientes apresentaram lesões neoplásicas ou pré-malignas que não seriam diagnosticados se fosse utilizada apenas sigmoidoscopia.

TABELA 1 - Diagnósticos histológicos das lesões suspeitas de neoplasia

\begin{tabular}{|c|c|c|c|c|c|}
\hline & \multicolumn{2}{|c|}{ Adenocarcinomas } & \multirow[t]{2}{*}{ Adenomas } & \multirow[t]{2}{*}{ Outros } & \multirow[t]{2}{*}{ Total } \\
\hline & Proximais & Distais & & & \\
\hline $\mathrm{n}$ & 6 & 20 & 10 & 2 & 38 \\
\hline$\%$ & 15,7 & 52,6 & 26,3 & 5,2 & 100 \\
\hline
\end{tabular}

TABELA 2 - Histologia das lesões polipóides

\begin{tabular}{lcc}
\hline & Quantidade & \% \\
\hline Adenoma & 48 & 68,5 \\
Adenocarcinoma & 5 & 7,1 \\
Pólipo hiperplásico & 7 & 10 \\
Processo inflamatório & 8 & 11,4 \\
Tecido de granulação & 1 & 1,4 \\
Normal & 1 & 1,4 \\
Total & 70 & 100 \\
\hline
\end{tabular}

TABELA 3 - Histologia dos pólipos adenomatosos

\begin{tabular}{lccc}
\hline & Proximal & Distal & Total \\
\hline Tubular & 11 & 15 & 26 \\
Túbulo-viloso & 12 & 19 & 31 \\
Viloso & 0 & 1 & 1 \\
Total & 23 & 35 & 58 \\
\hline
\end{tabular}


TABELA 4 - Histologia de todas as lesões proximais

\begin{tabular}{lcc}
\hline Histologia & Lesões isoladas & Total \\
\hline Adenocarcinoma & 7 & 10 \\
Linfoma & 0 & 1 \\
Adenomas & 16 & 23 \\
Pólipo hiperplásico & 2 & 4 \\
Pólipo inflamatório & 2 & 6 \\
Tecido de granulação & 1 & 1 \\
Total & 28 & 45 \\
\hline
\end{tabular}

\section{DISCUSSÃO}

Atualmente está bem estabelecida a importância da transformação adenoma para adenocarcinoma na gênese do câncer colorretal e que a polipectomia é método eficaz na sua profilaxia ${ }^{(9,12,13,14,15,16)}$.

As recomendações aceitas para rastreamento do CCR incluem pesquisa de sangue oculto anual e sigmoidoscopia a cada três ou cinco anos, ambos a partir dos 50 anos, para pacientes de risco médio, e mais precocemente para vigilância de pacientes de alto $\operatorname{risco}^{(1,2,3)}$. Argumenta-se que o pequeno número de lesões proximais não justifica o aumento do desconforto, risco e custo, para a realização de colonoscopia no lugar da sigmoidoscopia.

A sigmoidoscopia é de fácil execução, barata, causa pouco desconforto durante o preparo e execução. A colonoscopia, apesar de mais cara e desconfortável, é o método mais sensível para detectar pólipos em todo o cólon, com baixas taxas de falso-positivo e negativo. Além disto, tem a vantagem de ser terapêutico durante o mesmo procedimento, quando comparado ao enema opaco e colonoscopia virtual $^{(12)}$. Assim sendo, começam a surgir dúvidas a respeito das recomendações tradicionais, sugerindo-se o uso rotineiro da colonoscopia para o rastreamento.

$\mathrm{REX}^{(10)}$, estudando 900 voluntários assintomáticos e com pesquisa de sangue oculto negativo, concluiu que idade e sexo masculino foram os únicos fatores preditivos de adenomas e que a colonoscopia identificou o dobro de lesões em relação à retossigmoidoscopia. Propõe que uma colonoscopia única aos 60 anos seria suficiente para rastreamento eficaz.

ROGGE et al. ${ }^{(12)}$ sugeriram colonoscopia sem pesquisa de sangue oculto a cada 5 anos para rastreamento do CCR. Segundo esses autores, tal método teria custo semelhante ao rastreamento do câncer de mama e de colo de útero, com eficácia superior aos métodos tradicionais.
THIIS-EVENSEN et al. ${ }^{(13)}$, estudando idosos assintomáticos, concluíram que $54 \%$ dos adenomas desta população eram proximais mas, devido a idade avançada desta população, não haveria tempo suficiente para a evolução para adenocarcinoma. Na amostra desta série, $39,65 \%$ dos adenomas eram proximais, sendo que $27,58 \%$ eram isolados.

KADAKIA et al. ${ }^{(6)}$, estudando pacientes com risco médio e pesquisa de sangue oculto negativo, encontraram adenomas proximais em $23 \%$ dos pacientes sem alterações distais e em $29 \%$ daqueles com pólipos distais, concluindo que adenomas acometem um quarto da população de médio risco, assintomática, com pesquisa de sangue oculto negativo, independente da presença de adenomas distais. Esta taxa foi semelhante em nosso estudo $(27,58 \%)$.

Em outro estudo, REX et al. ${ }^{(11)}$ investigaram 116 pacientes com CCR proximal ao angulo esplênico, todos com risco médio. Dentre eles, 58,6\% tinham sigmoidoscopia normal.

IMPERIALE et al. ${ }^{(4)}$ estudaram adultos assintomáticos com idade igual ou superior a 50 anos que foram submetidos a colonoscopia, avaliaram a relação de lesões de risco no cólon proximal associadas ou não a lesões do cólon distal, concluindo que se a colonoscopia fosse feita apenas em pacientes com pólipos com cólon distal, a metade dos pacientes com lesão de risco no cólon proximal não seriam detectados.

No presente estudo encontraram-se $22,77 \%$ de lesões com importância clínica no cólon proximal, que não seriam diagnosticadas pela sigmoidoscopia. Por outro lado, 30,43\% dos pacientes com adenomas distais tiveram adenomas proximais. Isto indica que, embora um em cada três pacientes submetidos a sigmoidoscopia, contendo adenomas em cólon proximal, seria encaminhado à colonoscopia, dois em cada três permaneceriam com lesões de risco não diagnosticadas.

Este estudo pode ser criticado por não se excluírem os pacientes com neoplasia, que poderiam ter sangue oculto positivo, sendo detectados em rastreamento normal. De qualquer forma, a pesquisa de sangue oculto falso-negativa levaria à perda de diagnóstico nestes pacientes com lesões proximais, que são em número considerável.

Embora os dados apresentados neste estudo permitam discutir os protocolos atuais de rastreamento, são necessários estudos prospectivos para confirmar se a colonoscopia isolada diminuirá a mortalidade do CCR e se de fato a relação custo-benefício é positiva.

No futuro a perspectiva de uso rotineiro da colonoscopia virtual, ainda que sem possibilidade terapêutica, terá a vantagem de diminuir significativamente o desconforto do paciente e poderá ser de uso rotineiro para o rastreamento do câncer colorretal. Até que a colonoscopia virtual tenha seu papel melhor estudado, sugere-se o uso da colonoscopia para rastreamento do câncer colorretal. 
Nakao FS, Cury MS, Ferrari Jr AP. Colonoscopy or flexible sigmoidoscopy: risk of isolated right colon lesions. Arq Gastroenterol 2001;38(3):168171.

ABSTRACT - Background - Colorectal cancer is an important cause of death in western countries. Screening methods are based on flexible sigmoidoscopy, a cheap, effective, and less painful procedure, but some important lesions on the right colon can be missed. Aim - Evaluate how many important lesions would be missed if colonoscopy indicated only for patients with distal lesions identified during flexible sigmoidoscopy. Material and Methods - All consecutive colonoscopy performed in the Endoscopy Unit of the Gastroenterology Division of Federal University of São Paulo, SP, Brazil, with polyps and cancer suspicious lesions were reviewed. Patients with a second procedure, inflammatory bowel disease or colonic surgery were excluded. Results - We reviewed 101 patients with 38 possible cancer lesions and 70 polyps. The mean age was 62 years (SD 13,7 years) and 45 were male. Forty five lesions were in the right colon, namely 23 adenomas, 10 adenocarcinomas, 1 lymphoma and 11 benign lesion. Among such lesions, 28 were isolated in the right colon (16 adenomas and 7 adenocarcinomas). Conclusion - In our study 23 patients (22,77\%) had adenomas or adenocarcinomas isolated in the right colon, that would be missed if flexible sigmoidoscopy was used alone.

HEADINGS - Colonoscopy. Sigmoidoscopy. Colonic polyps. Rectal neoplasms.

\section{REFERÊNCIAS BIBLIOGRÁFICAS}

1. Bond JH. Screening guidelines for colorectal cancer. Am J Med 1999;106:7S$10 \mathrm{~S}$.

2. DeCosse JJ. Early cancer detection. Colorectal cancer. Cancer 1988;62(8 Suppl):1787-90.

3. Helm JF, Sandler RS. Colorectal cancer screening. Med Clin North Am 1999;83:1403-22.

4. Imperiale TF, Wagner DR, Lin CY, Larkin GN, Rogge JD, Ransohoff DF. Risk of advanced proximal neoplasms in asymptomatic adults according to the distal colorectal findings. N Engl J Med 2000;343:169-74.

5. Instituto Nacional do Câncer, Rio de Janeiro. Estimativa de incidência e mortalidade por câncer no Brasil para 1999. Rio de Janeiro: INCA; 1999. www.inca.org.br, www.sidra.ibge.gov.br .

6. Kadakia SC, Wrobleski CS, Kadakia AS, Meier NJ. Prevalence of proximal colonic polyps in average-risk asymptomatic patients with negative fecal occult blood tests and flexible sigmoidoscopy [see comments]. Gastrointest Endosc 1996;44:112-7.

7. Kronborg O. Follow-up after removal of colorectal adenomas and radical surgery for colorectal carcinomas. Br J Surg 1985;72 Suppl:S26-8.

8. Lefton HB, Pilchman J, Harmatz A. Colon cancer screening and the evaluation and follow-up of colonic polyps. Prim Care 1996;23:515-23.

9. Morson BC, Bussey HJ. Magnitude of risk for cancer in patients with colorectal adenomas. Br J Surg 1985;72 Suppl:S23-5.
10. Rex DK. Endoscopic screening for colorectal cancer: recent studies from Indiana University. Indiana Med 1994;87:68-73.

11. Rex DK, Chak A, Vasudeva R, Gross T, Lieberman D, Bhattacharya I, Sack E, Wiersema M, Farraye F, Wallace M, Barrido D, Cravens E, Zeabart L, Bjorkman D, Lemmel T, Buckley S. Prospective determination of distal colon findings in average-risk patients with proximal colon cancer. Gastrointest Endosc 1999;49:727-30.

12. Rogge JD, Elmore MF, Mahoney SJ, Brown ED, Troiano FP, Wagner DR, Black DJ, Pound DC. Low-cost, office-based, screening colonoscopy [see comments]. Am J Gastroenterol 1994;89:1775-80.

13. Thiis-Evensen E, Hoff GS, Sauar J, Majak BM, Vatn MH. Flexible sigmoidoscopy or colonoscopy as a screening modality for colorectal adenomas in older age groups? Findings in a cohort of the normal population aged 63-72 years. Gut 1999;45:834-9.

14. Watne AL. Colon polyps. J Surg Oncol 1997;66:207-14.

15. Williams CB. Polyp follow-up: how, who for and how often? Br J Surg 1985;72 Suppl:S25-6.

16. Winawer SJ, Zauber AG, Ho MN, O’Brien MJ, Gottlieb LS, Sternberg SS, Waye JD, Schapiro M, Bond JH, Panish JF. Prevention of colorectal cancer by colonoscopic polypectomy. The National Polyp Study Workgroup [see comments]. N Engl J Med 1993;329:1977-81.

Recebido em 26/9/2000 Aprovado em 6/2/2001. 